\title{
EXPERIENCE RATING \\ A NEW APPLICATION OF THE COLLECTIVE THEORY OF RISK *)
}

\author{
Hans Ammeter \\ Zurich (Switzerland)
}

Besides its well known applications, the collective risk theory has recently also been applied to problems connected with the so called Experience Rating. This term is used to define a method of premium calculation in insurance business which is based partially or totally on the individual experience of the particular risk involved. It is obvious that Experience Rating is essentially applicable to collective insurances which contain no saving element. In practical applications various possibilities may be considered.

The collective theory of risk provides an efficient calculus for the analysis of the various forms of Experience Rating and in paper [3] ${ }^{* *}$ ) a particular form of Experience Rating for collective insurances is examined. It is there assumed that the collective risk premium is based on experience derived from non-individual observations. If any cost loading is disregarded, the net premium is given by the relation $P^{\prime}=(\mathrm{r}+\lambda) P$, where $\lambda$ is a security factor and $P$ the part of the premium covering the expected claims cost. A premium refund

$$
G=\alpha^{\prime} P^{\prime}-\beta S
$$

is to be deducted from the basic net premium $P^{\prime}$. In this formula $\alpha^{\prime}$ and $\beta$ are suitable numerical values and $S$ means the due sum to be paid out for claims. Hence the net cost to the group considered depends on the actual claims $S$ and therefore takes into account the individual claims experience. It may be shown that for $\beta=\mathrm{I}$ the form of Experience Rating considered is equivalent to a stop loss cover. The general case with $\beta \neq \mathrm{I}$ represents a combination of ordinary insurance cover and stop loss cover.

*) Paper presented to the Rättvik Colloquium I96I.

**) Numbers in [] refer to the references at the end of the present paper. 
I.

In paper [3] the determination of a premium refund according to formula (I) is investigated, i.e. the numerical values of $\alpha^{\prime}$ and $\beta$ are found by taking into consideration the essential risk properties of the group and starting, in particular, from the following assumptions:

a) The cost of claims $S$ is a random variable distributed according to the generalized Poisson law corresponding to the usual assumptions in the collective risk theory. In order to facilitate numerical computations the Poisson distribution is approximated by a normal distribution with equal mean and variance.

b) The parameters $\alpha^{\prime}$ and $\beta$ in formula ( $\mathrm{I}$ ) are determined so that an unbiased premium refund is obtained, i.e. the mean value $E(G)$ of the premium refund $G$ is equal to the margin $\lambda P$ contained in the tariff. An unlimited number of pairs $\alpha^{\prime}$ and $\beta$ exists which satisfy this condition. Therefore one parameter may be arbitrarily chosen, the other one then being completely determined. Further details are given in paper [3].

The question arises as to the extent of the errors introduced by the approximation by means of the normal distribution to the numerical values of the parameters $\alpha^{\prime}$ and $\beta$. In order to clear up this matter the numerical examples of paper [3] have been computed with the underlying Poisson distribution. The same calculation have also been carried out under the assumption of a negative binomial distribution taking into account unstable risk rates, etc.

In table I the values listed in the first column of parameter $\alpha^{\prime}$ of a premium refund according to formula ( $I$ ) were arbitrarily chosen and corresponding values of $\beta$ determined individually for each distribution on the basis of an unbiased refund. The values of the parameter $\beta$ differ for each distribution and are based in particular on the following assumptions:

$A$. Instead of $S$ the standardized auxiliary variable

$$
z=\frac{S-t}{\sigma(S)}
$$

is introduced, which is assumed to be normally distributed with zero mean and unit standard deviation. To simply matters it is 
assumed that claims are equal in amount. In this case the expected amount of claims $S$ is identical with the expected number of claims $t$ and for the standard deviation

$$
\sigma(S) \text { the relation } \quad \sigma(S)==\sqrt{t} \text { holds. }
$$

$B$. Assuming equal sums to be paid out for claims it is sufficient to consider only the actual number of claims $r$, for which a Poisson distribution will hold

$$
f(r)=\frac{e^{-\imath} t^{r}}{r !}
$$

$C$. The actual number of claims $r$ is assumed to be distributed according to a negative binomial law

$$
f(r)=\left(\frac{h+r-\mathbf{I}}{r}\right) \cdot\left(\frac{t}{t+h}\right)^{r} \cdot\left(\frac{h}{t+h}\right)^{h}
$$

The fluctuation parameter $h$ in formula (3) decreases with growing basic variance. In the limit as $h \rightarrow \infty$ the distribution (3) reduces to the Poisson distribution (2). In the examples given below the value $h=\mathrm{I} 6$ was assumed, corresponding to a basic standard deviation of the claim rates of $25 \%$.

\section{TABLE I}

\begin{tabular}{|c|c|c|c|c|}
\hline \multirow{3}{*}{$\alpha^{\prime}$} & \multicolumn{4}{|c|}{$\beta$} \\
\hline & \multicolumn{4}{|c|}{ Assumption } \\
\hline & $A$ & $B$ & $C$ & $A^{\prime}$ \\
\hline 28,074 & 95,479 & $75, \mathrm{I} 3 \mathrm{I}$ & I0 7,443 & I 901,462 \\
\hline 5,203 & 9,512 & 9,085 & 10,940 & 19,832 \\
\hline 1,586 & $I, 983$ & 1,979 & $2,18 I$ & $2,4^{1} 5$ \\
\hline 0,769 & 0,730 & 0,734 & 0,767 & $0,77^{8}$ \\
\hline 0,514 & 0,394 & 0,394 & 0,400 & 0,400 \\
\hline 0,4 I I & 0,263 & 0,263 & 0,265 & 0,265 \\
\hline $0,35^{8}$ & 0,198 & $0, \operatorname{Ig} 8$ & 0,198 & 0,198 \\
\hline 0,305 & 0,132 & 0,132 & 0,132 & 0,132 \\
\hline 0,263 & 0,079 & 0,079 & 0,079 & 0,079 \\
\hline 0,206 & 0,008 & 0,008 & 0,008 & 0,008 \\
\hline 0,200 & o & o & o & 0 \\
\hline
\end{tabular}

Numerical values of the parameters $\alpha^{\prime}$ and $\beta$ for a premium refund under several assumptions of the distribution of the amount $S$ to be paid for claims. 
$A^{\prime}$. The values of $\beta$ in this case were computed under the assumption of a normal distribution identical in mean and variance with the negative binomial distribution $C$.

The normal distributions $A$ and $A^{\prime}$ are truncated to avoid negative values of claims $S$. The range of negative sums is related to the damage free case. All numerical examples are based on an expected number of claims $t=$ Io, equal sums to be paid out for claims and a security loading of $\lambda=0,25$.

A comparison of the various numerical values of $\beta$ in the table on page 5 gives rise to the following remarks:

For moderate values of $\alpha^{\prime}$ the corresponding values of $\beta$ are approximately the same for the four types of distributions considered. Significant differences only occur for large values of the parameter $\alpha^{\prime}$, where the weight of the sums to be paid for claims is comparatively high. In paper [3] it is shown that large values of the parameters $\alpha^{\prime}$ and $\beta$ lead to very unstable premium refunds, and are therefore of no practical importance. Though the examples dealt with illustrate only a small range of cases which might be considered it is surprising how often the normal approximation leads to useful results in practise.

II.

Experience Rating is a valuable technique, if it is feared that the assumptions underlying the calculation of the basic net premium $P^{\prime}$ are inaccurately based so that in particular cases a poor approximation might occur. The inaccuracy of $P^{\prime}$ will be more or less compensated by the premium refund $G$. The consequences of such poor approximation to $P^{\prime}$ will next be considered at first from the point of view of the insurer.

Although such poor approximations may occur individually, it might nevertheless be assumed that the portfolio of the insurer considered as a whole is rated adequately, so that the expected number of claims is in agreement with the underlying assumptions. On the other hand it may be assumed that in a particular group the expected number of claims is $t q$, different from the number $t$ corresponding to underlying assumptions in the calculation of $F^{\prime}$; 
the factor $q$ is assumed to be a random variable distributed according to the $\Gamma$ - Law

$$
d H(q)=\frac{h^{h} e^{-h q} q^{h-1}}{\Gamma(h)} d q
$$

with unit mean and variance $h^{-1}$.

As shown by the authcr in previous papers e.g. [I], the Poisson distribution (2) changes under these assumptions into the negative binomial distribution (3). The assumption of divergent claim rates therefore requires the substitution of the Poisson distribution by the negative binomial law. Although this assumption may represent only a rough approximation to the real situation, it certainly gives better results than the assumption of uniform claim rates.

It is seen from the table I that assumption $C$ of a negative binomial distribution leads to larger values of the parameter $\beta$ for given $\alpha^{\prime}$ than for the assumption $B$ corresponding to homogeneous claim rates. Inverting this conclusion it is seen that the insurer would suffer a loss if he does not take into account the diversity of claim rates in the particular groups although it was assumed that the deviations from the basic risk level are mutually counterbalanced in the whole portfolio of the insurer. This apparent contradiction is clarified in the following way: For groups which are overrated the premium refund $G$ compensates the deviations from the real costs rather closely. On the other hand an insufficient premium can hardly be counterbalanced by a premium refund. An insurer should therefore be cautious and consider an adequate basic dispersion of the claim rates when he fixes the parameters $\alpha^{\prime}$ and $\beta$ for particular groups.

III.

In what follows the departure of claim rates from their expectation is discussed from the point of view of the insured group. This group expects to be rated correctly or, more precisely, the premium calculation should be unbiased for the individual claim experience of each single group. It is therefore necessary to investigate the consequences of poor approximations to $P^{\prime}$ on the expected value of the premium refund. 
Mean values of the premium refund $G$ as functions of the expected number of claims $t_{1}$

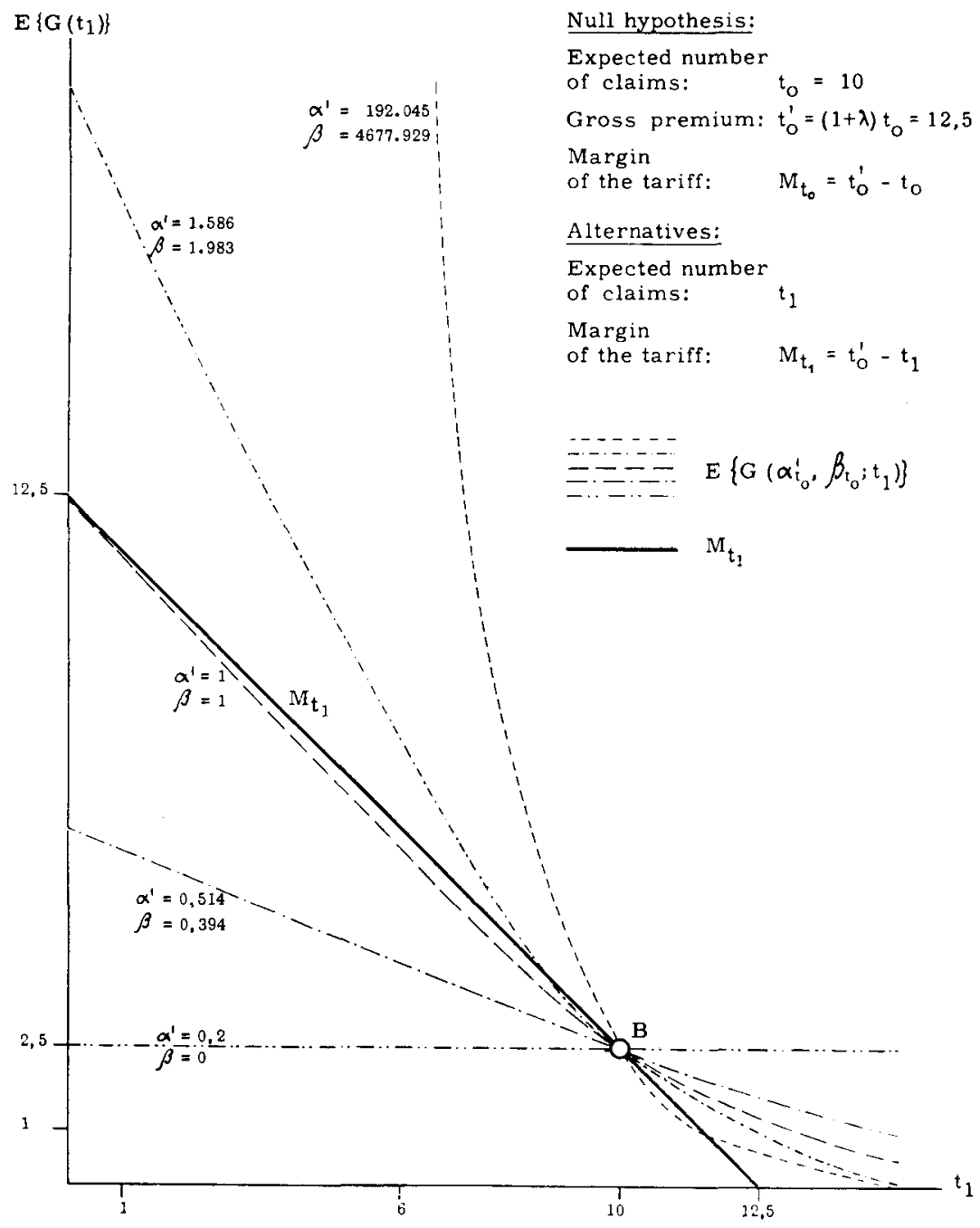


In the enclosed figure the mean values of the premium refund $G$ are given for example $(A)$ considered in the table I (expected number of claims $t_{0}=10 ; \lambda=0,25$; net premium with security loading $\left.t_{0}^{\prime}=(\mathbf{I}+\lambda) t_{0}=\mathbf{I} 2,5\right)$. In the figure the ordinates represent the mean value $E(G)$ of the premium refund and the abscissae the individual true number of expected claims $t_{1}$, which is supposed to differ from the number $t_{\mathbf{0}}$ because of poor approximation. The mean value $E(G)$ is assumed to be a function of the true expected number of claims of the group $t_{1}$. The pairs of parameters $\alpha^{\prime}$ and $\beta$, already used for this example, are unbiased with regard to the nullhypothesis (expected number of claims $t_{0}=10$ ). The lowest curve, which is horizontal, represents the pair of parameters $\alpha^{\prime}=0,2, \beta=0$. In this particular case the true claim level does not matter because $\beta=0$. All curves start for $t_{1}=0$ with the mean value $\alpha^{\prime} t_{0}^{\prime}$. With increasing $t_{1}$ the curves decrease and for $t_{1} \rightarrow \infty$ approach the $t_{1}$ - axis asymptotically. Since all pairs of parameters with regard to the null-hypothesis $t_{0}$ are unbiased, the whole set of curves meets in the cluster-point $B$ with coordinates $t_{0}, t_{n}^{\prime}-t_{0}$.

The broad straight line represents the true margin of the tariff $M_{t_{1}}=t_{0}^{\prime}-t_{1}$ corresponding to the assumed number of claims $t_{1}$ to be expected. The straight line also contains the cluster-point $B$ of the above mentioned set of curves. Points of intersection between curves of the mean value of the premium refund $G$ and the straight line $M_{t_{1}}$ show that the margin is in conformity with the expected value of the premium refund $G$, i.e. the formulae of the premium refund with the parameters $\alpha^{\prime}$ and $\beta$ are unbiased. The figure leads to the following conclusions:

For parameter values $\alpha^{\prime}<$ I the expectancy curves have only one point of intersection with the straight line $M_{t_{1}}$, the clusterpoint $B$. For values of $\alpha^{\prime} \geq \mathrm{I}$ there are usually two points of intersection with $M_{t_{1}}$. Hence it follows that two hypotheses always exist for the expected number of claims $t_{1}$ which lead to an unbiased premium refund. For $\alpha^{\prime}>$ I and with increasing $\alpha^{\prime}$ the points of intersection at first are left of the cluster point $B$, i.e. a second hypothesis $t_{1}<t_{0}$ will be unbiased besides the null-hypothesis $t_{1}=t_{0}$. Above a certain critical value of $\alpha^{\prime}$ the second point of intersection appears for $t_{1}>t_{0}$, i.e. besides the null-hypothesis a second hypothesis with $t_{1}>t_{0}$ leads also to unbiased results. Hence it may be concluded 
that a combination of $\alpha^{\prime}$ and $\beta$ can be found in general, for which the null-hypothesis and a certain counter-hypothesis in the range $t_{1}<t_{0}^{\prime}$ is valid, on the other hand only pairs of parameters $\alpha^{\prime}$ and $\beta$ with $\alpha^{\prime}>$ I are in agreement with two underlying hypotheses.

An ideal solution would be obtained if a combination of $\alpha^{\prime}$ and $\beta$ could be found for which the mean value of the premium refund $G$ would follow the margin line $M_{t_{1}}$ in the whole range $t_{1} \leq t_{0}$. If such a pair of parameters could be found a uniformly unbiased premium refund $G$ would be reached. A closer study shows that this postulate is approximately realized for $\alpha^{\prime}=\beta=\mathrm{I}$ and that the approximation improves with increasing margin $\lambda t_{0}$ relative to the null-hypothesis $t_{0}$. It follows that for a sufficiently large margin a uniformly unbiased Experience Rating can be stated with the simplified formula for a premium refund $G=P^{\prime}-S$. However this basis of calculation has the disadvantage that in general the margin $M_{t_{0}}=t_{0}^{\prime}-t_{0}$ has to be chosen so high that the insurer covers only a very modest risk.

Further conclusions may be drawn from the graph e.g. the more the insurer really covers a risk the less will the Experience Rating formula be unbiased. Moreover it is seen that premium refunds with $\alpha^{\prime}>$ I which comply exactly with two hypotheses about the individual margin $M_{t_{1}}$, are only reasonable within a relatively small range around $t_{0}$. Outside this range the mean value $E(G)$ falls far beyond acceptable limits.

A remarkable peculiarity may be derived from the graph, i.e. when the margin line $M_{t_{1}}$ touches the appartaining expectancy curve in the cluster-point $B$. In this special case the expected value of the premium refund is never smaller than the margin of the tariff $M_{t_{1}}$, so that for any counter-hypothesis $t_{1}$-excluding the null-hypothesis-the insurer has to expect a deficit. For this tangent constellation the factors $\alpha^{\prime}$ and $\beta$ are always larger than $\mathbf{I}$. For increasing $t$ and increasing margin the refund parameters tend towards the afore-mentioned special case $\alpha^{\prime}=\beta=\mathrm{I}$.

\section{IV}

As a rule the application of a premium refund formula with two parameters allows to base the determination of the parameters 
on two conditions. It is therefore possible to denote two conditional equations which lead from a certain point of view to optimal solutions. For example the following three optimal solutions. are possible:

\section{I) Stability and unbiased experience rating}

As a rule an unbiased refund for any counter-hypothesis is granted the more the larger the parameter $\beta$ is chosen. Extremely large $\beta$ may however not lead to a satisfactory solution since the premium refund will fluctuate in such a way that the equalization principle becomes illusory. An optimum satisfying both aspects - stability and unbiased experience rating-may be found by starting from a highest value of $\beta$ still in accord with the insurance principle, $\alpha^{\prime}$ being rated according to the null-hypothesis. This method is to be recommended for small groups with a small security margin, whereas for large groups stability is granted to a certain extent in advance.

\section{2) Experience Rating uniformly unbiased in the interval $t_{1} \leq t_{0}$}

Chapter III has shown that the peculiar parameter constellation $\alpha^{\prime}=\beta=$ I leads to a continuously unbiased premium refund in the counter-hypothetic interval $t_{1} \leq t_{0}$. The selection of these special parameters results in an optimally unbiased experience rating in the relevant interval. Numerical computations show however that the application of this method proves only useful for large groups.

\section{3) Experience Rating uniformly unbiased in the local interval $t_{\mathbf{0}} \pm t_{\mathbf{1}}$}

Rather often the real expected value $t_{1}$ is uncertain but only in a locally limited interval in the surroundings of the null-hypothesis $t_{0}$. It would thus be desirable to find a parameter constellation for which a continuous unbias in the proximity of the null-hypothesis would be granted. This aim is achieved in the following way:

In chapter III the special case was mentioned where the expectancy curve touches the margin line $M_{t_{1}}$ in the cluster-point $B$ with the coordinates $\left(t_{0}, t_{0}^{\prime}-t_{0}\right)$. In this particular tangent constellation the expectancy curve undoubtedly approaches the most the margin line in the proximity of $t_{0}$. One could therefore start from this 
tangent constellation and add a small security margin to prevent a loss to the insurer for every counter-hypothesis. The most efficient procedure would consist in raising the parameter $\beta$ adequately in such a way that the expectancy curve would be a little shifted in comparison with the tangent curve and approach the margin line sufficiently close in the preselected interval.

For further details reference is made on paper [4].

\section{REFERENCES}

[I] Ammeter, H.: Die Ermittlung der Risikogewinne im Versicherungswesen auf risiko-theoretischer Grundlage. ,Mitteilungen der Vereinigung schweizerischer Versicherungsmathematiker", 57. Band, Heft 2.

[2] A Rational Experience Rating Technique for Group Insurance on the Risk Premium Basis. Proceedings of the XVth International Congress of Actuaries, New York I957.

[3] Stop Loss Cover and Experience Rating. Proceedings of the XVIth International Congress of Actuaries. Brussels 1960.

[4] Risikotheoretische Grundlagen der Erfahrungstarifierung. ,,Mitteilungen der Vereinigung schweizerischer Versicherungsmathematiker", 6r. Band, Heft 2. 